14 Ravindran RS, Turner MS, Muller I. Neurological effects of subarachnoid administration of 2-chloroprocaine-CE, bupivacaine and low $\mathrm{pH}$ normal saline in dogs. Anesth Analg 1982; 61: 279-83.

15 Wong BC, Spiedholz NI, Hillman DE, Turndorf $H$. Subarachnoid sodium bisulfite (the antioxidant in Nesacaine) causes chronic neurological deficit. Aneschesiology 1982; 57: A194.

16 Rosen MA, Baysinger CL, Shnider SM et al. Evaluation of neurotoxicity of local anesthetics following subarachnoid injection. Anesthesiology 1982; 57: A196.

\title{
Neurotoxicité potentielle des agents anesthésiques locaux
}

L'agent anesthésique local 2-chloroprocaine (Nesacaine) a été récemment introduit au Canada alors qu'il était disponible aux Etats-Unis depuis plusieurs années. La chloroprocaine est un agent anesthésique local amino ester dont la structure est similaire à celle de la procaine. Son action est rapide et sa durée d'anesthésie relativement courte. Son avantage primordial en clinique est qu'il semble avoir moins de toxicité systémique à cause de son hydrolyse rapide par la cholinestérase du plasma. Récemment, la neurotoxicité potentielle de la chloroprocaine a été un sujet d'intérêt considérable aux Etats-Unis.

La neurotoxicité des agents anesthésiques locaux intéresse les anesthésistes depuis plusieurs annees. Quoique des rapports sporadiques sur les réactions locales soient apparus dans des articles médicaux, d'amples études d'épidémiologie ont indiqué que l'emploi de l'anesthésie régionale est une procédure remarquablement inoffensive lorsqu'exécutée correctement. ${ }^{1,2}$ Le plus grand intérềt concernant la neurotoxicité a trait à l'usage intra-dural des agents anesthesiques locaux puisque cet usage peut conduire à des effets catastrophiques produits par des lésions de la moëlle épinière chez un patient sain. Cependant des études sur l'épidémiologie de l'anes- thésie rachidienne s'intéressant particulièrement à la tétracaine et à la lidocaine indiquent que cette technique est remarquablement sans danger. ${ }^{3-5} \mathrm{Par}$ contre il est connu que des agents anesthésiques locaux sont susceptibles de causer une irritation locale et une neurotoxicité atteignant des concentrations habituellement plus fortes que celles employées en clinique. 6

L'intérêt porté au potentiel de neurotoxicité des anesthésiques locaux après l'administration intradurale a refait surface aux Etats-Unis suite à quatre cas de deficits sensoriel/moteur prolongés reliés semble-t-il à l'injection sous-arachnoidienne accidentelle de chloroprocaine. ${ }^{7,8}$ Les éléments suivants nous ont obligés a porter notre attention sur ces quatre cas: 1) Chacun des quatre cas se présenta au cours d'un intervalle de six mois en 1979-1980. 2) Trois cas se présentèrent dans une institution en Indiana alors que le quatrième cas se présenta en Californie. 3) La plupart des cas semblent être à la suite d'injections intra-durales accidentelles de fortes doses de chloroprocaine qui étaient censées être employées pour l'administration dans l'espace Épidural. 4) Toutes les patientes étaient des jeunes femmes en santé sans antécédents d'abnormalité neurologique. 5) Une similarité semble exister entre 
la dose de chloroprocaine administrée et la sévérité des altérations neurologiques (Tableau I). Suite à ces rapports, on a observé cinq autres cas de neurotoxicité apparente après l'usage de chloroprocaine en $1982 .{ }^{\circ}$ Cependant, ces cas se manifestisrent au cours d'une période de plusieurs années et ne semblent pas avoir de rapport avec les cas rapportés initialement. De plus, la bupivacaine et la chloroprocaine furent administrées à plusieurs de ces patients.

Un éditorial publié en 1980 a essayé d'analyser le problème dans son éventualité. ${ }^{10}$ Se basant sur les informations connues à cette date, on en tire ces conclusions: 1) les réactions neurotoxiques sont causées par l'usage inopportun de la chloroprocaine; 2) la neurotoxicité potentielle de fortes doses de chloroprocaine administrées par voie intradurale ne peut pas être exclue, 3) le pH des solutions commerciales de la chloroprocaine est significativement plus bas que celui d'autres agents anesthésiques locaux et a pu contribuer à ces effets adverses. Il a été recommandé de poursuivre des études additionnelles chez des animaux afin de clarifier la neurotoxicité potentielle de la chloroprocaine. Cepenclant, l'usage d'une dose d'essai adéquate et l'administration de doses fractionnées d'agents anesthésiques locaux furent recommandés, si possible, en vue d'éviter les accidents que causerait l'administration sous-arachnoidienne de larges doses d'agents anesthésiques locaux. Finalement, il est à suggérer, si possible, que le liquide céphalorachidien soit obtenu après l'administration intradurale connue de larges doses d'agents anesthésiques locaux afin de diminuer la concentration de l'anesthésique tocal du liquide céphalo-rachidien.

Le rapport de ces cas a engendré un intérêt considérable pour la toxicité potenticlle de la chloroprocaine dans un climat de controverse et d'émotion. Les études scientifiques qui ont été faites pour clarifier cette question sont malheureusement contradictoires. De plus, jusqu'à maintenant très peu de ces études ont été publiées. Celles qui l'ont été sont résumées dans le Tableau II. Deux études ont examiné les réactions locales de tissus sur les nerfs périphéraux exposés à plusieurs agents anesthésiques locaux. Barsa et al. ont observé des effet: irritants plutôt sévères après que le nerf vague du lapin fut exposé à la chloroprocaine, mais non pas à la lidocaine ou à la bupivicaine. " D'autre part, Pizzolato et Reneger n'ont pas observé de changements histologiques aux nerfs sciatiques du rat exposés à la chloroprocaine et à la lidocaine pendant une période de six heures. ${ }^{12}$

Plusieurs études in vivo ont été faites sur des animaux recevant par voie intra-durale plusieurs agents anesthésiques locaux. Des doses intradurales ( $1 \mathrm{ml}$ ) chez les chiens auxquels on a administré plusieurs agents anesthésiques locaux incluant la chloroprocaine n'ont pas causé d'effets toxiques manifestes. ${ }^{13}$ Cependant, l'administration sous-arachnoidienne de larges doses de chloroprocaine chez les chiens a causé la paralysie chez 35 pour cent des animaux traités. ${ }^{14}$ Les chiens traités avec une dose similaire de bupivacaine n'ont pas manifesté de paralysie. De plus, l'injection sousarachnoidienne de sérum physiologique normal au pH de 3.0 n'a eu aucun effet.

Chez les lapins, des solutions commerciales de chloroprocaine causèrent la paralysie quand elles furent administrées en doses de 16 a $20 \mathrm{mg}$ par voie intra-durale alors que des solutions de chloroprocaine sans préservatif ne causèrent pas de paralysie. L'administration de sodium bisulfite, l'antioxydant dans les solutions commerciales de chloroprocaine, engendra chez les lapins une paralysie permanente. ${ }^{15}$

Une étude approfondie a été faite chez les moutons soumis à l'anesthésie rachidienne totale avec la chloroprocaine, la lidocaine, la bupivacaine, ou d'autres solutions de contrôle. ${ }^{16}$ Quoiqu'on ait observé des signes de neurotoxicité chez certains moutons, il n'y eut pas de nettes différences en neurotoxicité entre les agents anesthésiques locaux et les solutions de contrôle. Des études préliminaires faites chez huit singes n'ont révélé aucun signe de paralysie après l'anesthésie rachidienne totale soit avec la chloroprocainc ou la bupivacaine. L'évidence histologique de dimyélinisation a été observée dans la moëlle épinière de plusieurs singes avec les deux agents. ${ }^{16}$

En résumé, les preuves accumulées jusqu'à présent chez les animaux dans le but d'élucider le déficit sensoriel/moteur prolongé observé chez plusieurs patients après l'administration sous-arachnoïde de chloroprocaine sont contradictoires. Alors que plusieurs études ont démontré des déficits neurologiques suite à l'infusion de larges doses de chloroprocaine, d'autres études n'ont pas pâ confirmer ces observations. Une étude suggère que les changements neurologiques peuvent avoir un rap- 
port avec l'anti-bxidant sodium bisulfite qui est présent dans les solutions commerciales de chloroprocaine. Malheureusement, peu d'études ont été publiées en détail et sont insuffisantes pour permettre un examen scientifique minutieux qui pourrait détecter les raisons possibles pour ces contradictions. De plus, des études faites avec quatre espèces différentes peuvent indiquer des différences d'espèces dans la susceptibilité aux agents anesthésiques sur le tissu nerveux. Un facteur qui semble avoir été éliminé est le $\mathrm{pH}$ de la solution de chloroprocaine parce que les solutions de sérum physiologique normal au $\mathrm{pH}$ de $3.0 \mathrm{n}$ 'ont pas réussi à produire des déficits neurologiques. D'autres études sont indiquées afin de clarifier cette controverse.

En attendant d'avoir de plus amples informations, il serait prudent de prendre des précautions pendant l'operation du bloc épidural et d'utiliser une dose d'essai adéquate, afin d'éviter l'administration accidentelle intra-durale de larges doses de n'importe quel agent anesthésique local. Des doses fractionnées d'anesthésiques devraient être injectées, si possible, pour le bloc epidural. Quand de larges doses d'anesthésiques locaux ont été administrées par voie sous-arachnoïdienne, on devrait prélever approximativement $10 \mathrm{ml}$ de liquide céphalo-rachidien et le remplacer par un volume équivalent de sérum physiologique normal, lorsque l'aspiration du liquide céphalo-rachidien est possible, quoique l'efficacité de cette procédure n'est pas encore établie. Finalement, il y a peu à gagner et beaucoup à perdre en se servant d'accusations superficielles et de contre-accusations sur le danger de la chloroprocaine. Rien ne vaut les informations scientifiques et objectives. Personne sûrement n'est intéressé à détruire injustement un agent potentiellement utile ni à défendre imprudemment une substance potentiellement toxique. Nous esperons ne pas répéter l'histoire de l'anesthésie rachidienne de l'Angleterre où une génération de patients fut privé de cette technique d'anesthésie à cause d'inquiétudes sur la neurotoxicité résultant d'études mal documentées. Vu que la chloroprocaine est disponible seulement depuis peu au Canada, les chercheurs canadiens vont pouvoir assurer une évaluation plus objective du ratio nisque-bénéfice de cet agent particulier. 